\title{
Topology optimization for maximizing natural frequency separation
}

\section{Heitor N. Lopes*, Renato Pavanello}

\begin{abstract}
Resonance is a common problem in engineering. In order to avoid it, this work proposes a topology optimization method capable of obtaining a structure with a large range without natural frequencies around a given value.
\end{abstract}

\section{Key words: \\ Topology optimization, BESO, natural frequency}

\section{Introduction}

Topology optimization method has become of great importance in engineering problems and is used when designing new products. One of its applications can be to control a structure's natural frequencies, in order to satisfy certain design restrictions.

In this work, the method BESO (Bidirectional Evolutionary Structural Optimization) is used. This method is based on a sensitivity analysis, which is calculated by executing a finite element analysis.

\section{Results and Discussion}

The optimization problem a number of natural frequencies above and below a certain predefined value $\omega_{0}$ can be described as

$$
h\left(x_{i}\right)=\omega_{0}^{2}+\left[\frac{1}{M} \sum_{i=1}^{M} \frac{1}{\left(\omega_{n, i}^{2}-\omega_{0}^{2}\right)^{2}}\right]^{-\frac{1}{2}}
$$

here, $\omega_{0}$ is a prescribed frequency from which all natural frequencies are moved away and $M$ is the number of eigenvalues. It is assumed that the natural frequencies above the Mth one have little contribution to the sensitivity.

In conventional natural frequency optimization algorithms, some eigenvalues might approach each other, disturbing the convergence of the optimization algorithm, which is known as the mode shift problem. Since the proposed objective function determines the contribution of all eigenvalues, if two of them ever intersect, their sensitivities will smoothly transition.

Another common problem that may emerge is local modes, appearing due to the presence of islands of solid elements or thin bars in the structure during the optimization process. This is solved with a mode-tracking procedure, which compares the modes of each iteration with the latest one. That way, local modes are identified and removed from the optimization.

In order to analyze this method, a doubly clamped beam with dimensions of $150 \mathrm{~mm} \times 15 \mathrm{~mm} \times 15$ $\mathrm{mm}$ is studied. The domain was discretized in a $500 \times 50$ mesh of identical quadrilateral elements.

Its material is assumed to have a specific mass of $\rho=7800 \mathrm{~kg} / \mathrm{m}^{3}$, Young's modulus of $E=210 \mathrm{GPa}$ and Poisson's ratio of $v=0.3$. The BESO's parameters were: $E R=1 \%, A R_{\max }=2 \%, r_{\min }=2 \mathrm{~mm}, x_{\min }=10^{-6}$.

The final volume was fixed at $80 \%$ of the design domain and the operating frequency at $\mathrm{f}_{0}=40 \mathrm{kHz}$. Image
1 shows the evolution of some natural frequencies and topologies.

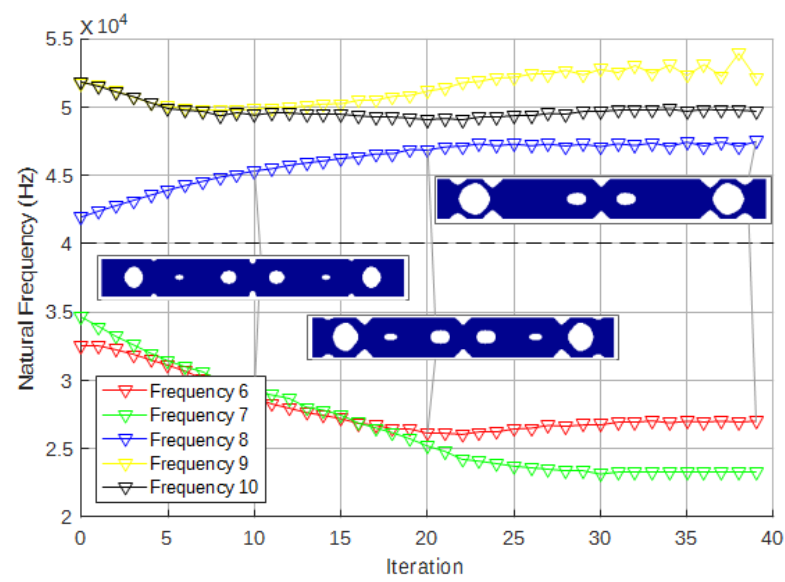

Image 1. Evolution of natural frequencies and obtained topologies.

The procedure successfully obtained a topology that moves natural frequencies away from the prescribed value. A decrease in the sixth and seventh natural frequencies is observed, as well as an increase in the eighth. Although the ninth and tenth ones experience a small decrease, their values stabilize as they approach the eighth, due to their contribution to the sensitivity.

\section{Conclusions}

This method of maximizing the separation between two adjacent natural frequencies was able to successfully obtain optimal topologies, which present significant gaps around the desired operating frequency.

This study can be extended and applied to other models, such as 3D ones, in order to obtain better results for different engineering applications.

\section{Acknowledgement}

The authors are grateful to CNPq for the financial support of this work.

\footnotetext{
${ }^{1}$ Huang, X. and Xie, M. Evolutionary topology optimization of continuum structures: methods and applications. New York: John Wiley \& Sons; 2010. $223 \mathrm{p}$.

2 Picelli, R., Vicente, W. M., Pavanello, R., \& Xie, Y. M. Evolutionary topology optimization for natural frequency maximization problems considering acoustic-structure interaction. Finite Elements in Analysis and Design, v. 106, p. 56-64, 2015. DOI: 10.19146/pibic-2017-78913
} 